\title{
Tender Soft Tissue Mass on the Base of the Neck
}

\author{
Ania Henning, MD; Stephen Wall, DO; Amy Deeken, MD; Silvia Verde de Peralta, MD
}

\section{Eligible for 1 MOC SA Credit From the ABD}

This Dermatopathology Diagnosis in our print edition is eligible for 1 self-assessment credit for Maintenance of Certification from the American Board of Dermatology (ABD). After completing this activity, diplomates can visit the ABD website (http://www.abderm.org) to self-report the credits under the activity title "Cutis Dermatopathology Diagnosis." You may report the credit after each activity is completed or after accumulating multiple credits.

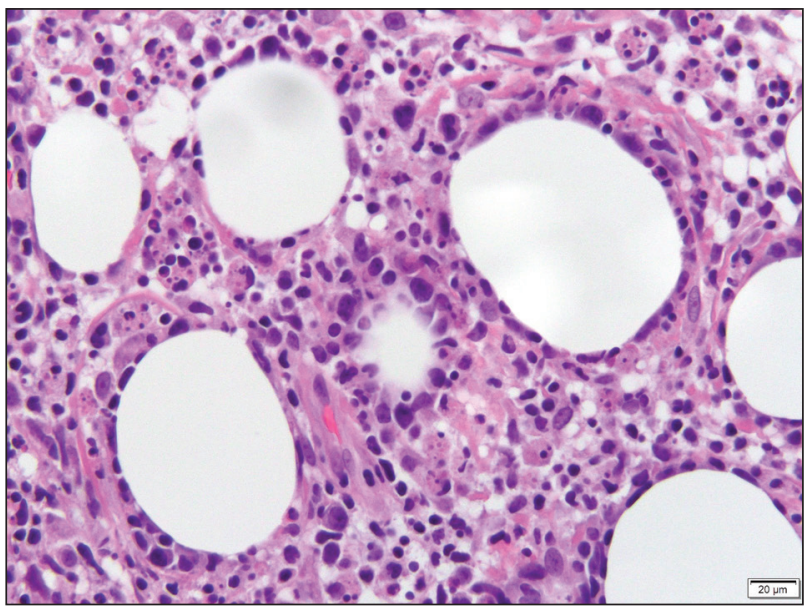

H\&E, original magnification $\times 400$. Reference bar indicates $20 \mu \mathrm{m}$.
A 47-year-old man presented with a tender soft tissue mass on the upper back with increasing discomfort over the last 4 weeks. He noted that he felt feverish a few times. Physical examination revealed a $3 \times 4-\mathrm{cm}$ area of induration involving the upper mid back with faint erythema of the overlying skin; no drainage was noted. A prominent left posterior cervical lymph node also was appreciated, and a punch biopsy of the mass was performed.

\section{THE BEST DIAGNOSIS IS:}
a. erythema nodosum
b. fat necrosis
c. lipodermatosclerosis
d. lupus erythematosus profundus
e. subcutaneous panniculitislike T-cell lymphoma

PLEASE TURN TO PAGE 27 FOR THE DIAGNOSIS

From the Department of Pathology \& Laboratory Medicine, Summa Health System, Akron, Ohio.

The authors report no conflict of interest.

Correspondence: Ania Henning, MD (aniahenning@gmail.com).

doi:10.12788/cutis. 0155 


\section{THE DIAGNOSIS: Subcutaneous Panniculitislike T-cell Lymphoma}

S ubcutaneous panniculitislike T-cell lymphoma (SPTCL) is a rare form of cutaneous lymphoma of mature cytotoxic $\mathrm{T}$ cells simulating panniculitis and preferentially infiltrating the subcutaneous tissue. ${ }^{1}$ Subcutaneous panniculitislike T-cell lymphoma can affect all ages but predominantly affects younger individuals, with $20 \%$ being younger than 20 years. ${ }^{2}$ It is a rare lymphoma that accounts for less than $1 \%$ of all non-Hodgkin lymphomas. ${ }^{3}$ It presents clinically as multiple subcutaneous masses, nodules, or plaques generally on the trunk or extremities. ${ }^{1,2}$ The skin surrounding the nodules may be erythematous, and the nodules may become necrotic; however, ulceration typically is not seen. Systemic symptoms such as fever, night sweats, and chills are present in half of cases. ${ }^{1}$ According to the World Health Organization, cytopenia and elevated liver function tests are common, and a hemophagocytic syndrome may be present in $15 \%$ to $20 \%$ of cases. ${ }^{3}$ The presence of a hemophagocytic syndrome yields a poor prognosis. ${ }^{1,3}$ Current guidelines denote that SPTCL T-cell receptor (TCR) $\alpha \beta$ is a distinct entity from the TCR $\gamma \delta$ phenotype, known as cutaneous $\gamma \delta$-positive T-cell lymphoma. ${ }^{3,4}$ Cutaneous $\gamma \delta$-positive T-cell lymphoma is associated with rapid decline and a worse prognosis. ${ }^{4}$

Histology of SPTCL is characteristic for a lobular panniculitislike infiltrate. ${ }^{1}$ The heavy subcutaneous lymphoid infiltrate is composed of atypical small- to medium-sized lymphocytes with mature chromatin and inconspicuous nucleoli lining adipocytes. The dense inflammatory infiltrate composed predominantly of neoplastic $T$ cells and macrophages may diffusely invade into the subcutaneous tissue. ${ }^{1}$ Admixed histocytes and karyorrhectic debris as well as rimming of the lymphocytes around the fat cells is typical and was seen in our patient (quiz image). The $\mathrm{T}$ cells of SPTCL have the following immunophenotype: TCR-beta $\mathrm{F}^{+}, \mathrm{CD}^{+}, \mathrm{CD} 4-\mathrm{CD}^{+}, \mathrm{CD}^{+} 6^{-}$. They can express numerous cytotoxic proteins, such as T1a-1, granzyme $\mathrm{B}$, and perforin., Although the $\mathrm{CD} 8^{+} \mathrm{T}$ cells may be sparse, they generally surround the adipocytes in a rimming manner and may distort the adipocyte membrane. ${ }^{1}$

Lupus erythematosus profundus (LEP) is a form of chronic cutaneous lupus that affects the deep dermis and fat. ${ }^{5}$ It also can present clinically as tender plaques or nodules. It most frequently involves the upper arms, shoulders, face, or buttocks - areas that are less commonly involved in other panniculitides. ${ }^{6}$ Histologically, LEP is similar to chronic discoid lupus with features such as epidermal atrophy, interface changes, and a thickened basement membrane (Figure 1). Lupus erythematosus profundus can present as a lobular panniculitis with mucin as well as a superficial and deep lymphocytic infiltrate that can involve the septa. ${ }^{5}$ Some cases of LEP have a predominantly lobular lymphocytic panniculitis in the absence of the typical epidermal or dermal changes of lupus erythematosus. Lymphoid follicles with germinal center formation are present in half of cases and reportedly are characteristic of LEP., ${ }^{6,7}$ The lymphoid follicles often have plasma cells, can extend into the septa as well as in between collagen bundles, and may have nuclear fragmentation. ${ }^{5}$ Another characteristic feature of LEP is hyaline sclerosis of lobules with focal extension into the interlobular septa. Immunofluorescence studies usually show linear deposition of IgM and C3 at the dermoepidermal junction. Antinuclear antibodies can be present in patients who have LEP but are not entirely specific. ${ }^{6}$

Lupus erythematosus profundus and SPTCL are part of a spectrum and may have overlapping clinical and histopathologic characteristics; therefore, distinguishing them may be difficult. $^{6-8}$ It is important to monitor these patients closely, as their disease may progress to lymphoma. ${ }^{6}$ Patients with SPTCL are more likely to present with advanced symptoms such as fever and hepatosplenomegaly and to succumb to hemophagocytic syndrome than patients with LEP.9

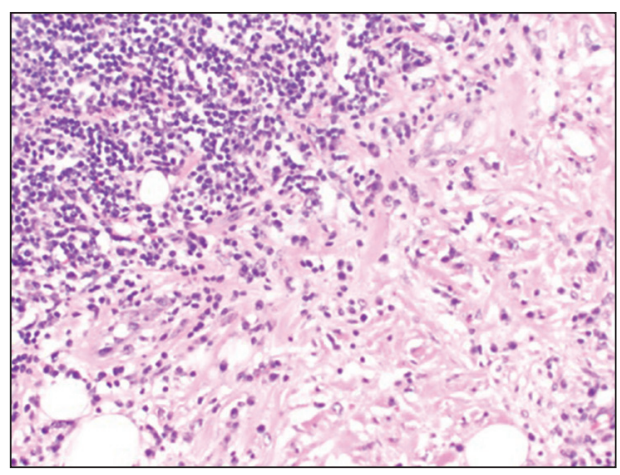

FIGURE 1. Lupus erythematosus profundus. A dense collection of lymphocytes in a lymphoid follicle with associated plasma cells (H\&E, original magnification $\times 40$ ).

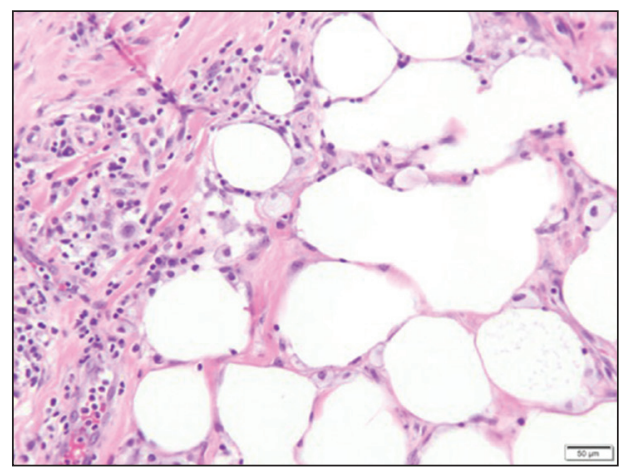

FIGURE 2. Fat necrosis. Lipid-laden macrophages along with chronic inflammatory cells (H\&E, original magnification $\times 200)$. Reference bar indicates $50 \mu \mathrm{m}$. 


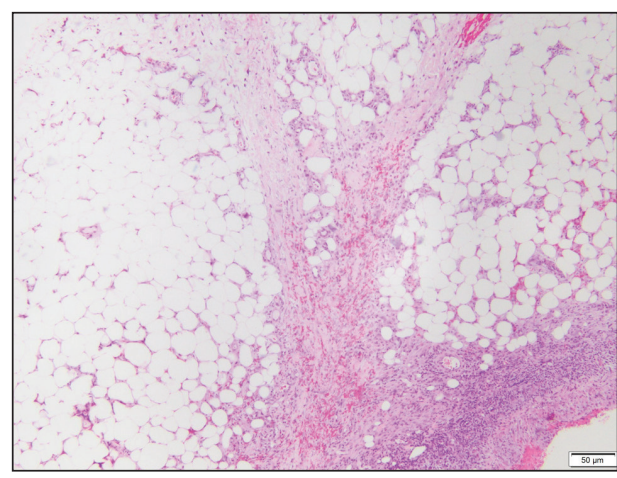

FIGURE 3. Erythema nodosum. Septal panniculitis with a mixed inflammatory background (H\&E, original magnification $\times 40$ ). Reference bar indicates $50 \mu \mathrm{m}$

Although SPTCL usually is clonal, several cases of LEP with clonality also have been described. Clonal LEP cases generally are identified in patients who present with fever and cytopenia. ${ }^{8}$ Lymphoid atypia and morphologic abnormalities may be seen in cases of LEP, further complicating the distinction between LEP and SPTCL. An elevated Ki67 level may be seen in cases of SPTCL with periadipocytic rimming. ${ }^{9}$ LeBlanc et al ${ }^{10}$ used Ki67"hot spots" along with CD8 immunohistochemistry to identify atypical lymphocytes associated with SPTCL. Lymphocyte rimming was defined by the presence of $\mathrm{CD} 8^{+}$lymphocytes with an elevated Ki67 index. Clinical, histopathologic, and molecular findings all should be used when dealing with challenging cases.

Fat necrosis can occur in any part of the body where trauma has occurred and can be associated with many disease processes. Patients typically present with a palpable mass, but a clinical history of trauma is not always present. Histopathologic findings include necrotic fat alongside lipid-laden foamy macrophages and scattered inflammatory cells (Figure 2). ${ }^{11}$ Fragments of normal as well as degenerating adipose tissue and multinucleated giant cells can be present.

Erythema nodosum (EN) is the most frequently encountered panniculitis and usually is seen in women in early adulthood. ${ }^{12}$ Patients present with several tender subcutaneous nodules and plaques that most commonly are present on the anterior surface of the legs. ${ }^{12,13}$ Patients may have a constellation of symptoms including fever and leukocytosis, but the disorder generally is self-limited. ${ }^{12}$ Erythema nodosum may be associated with a variety of diseases or infections including sarcoidosis, inflammatory bowel disease, and malignancy. ${ }^{14}$ The etiology of EN is diverse; therefore, a proper clinical workup may be necessary. Histopathology is that of a septal panniculitis with lymphocytes, histiocytes, and occasional eosinophils (Figure 3 ). ${ }^{13}$

Lipodermatosclerosis also occurs on the legs, most commonly in patients with venous insufficiency. ${ }^{12,15}$ Patients present clinically with pain, induration, redness, or swelling of the legs. Histopathology predominantly is

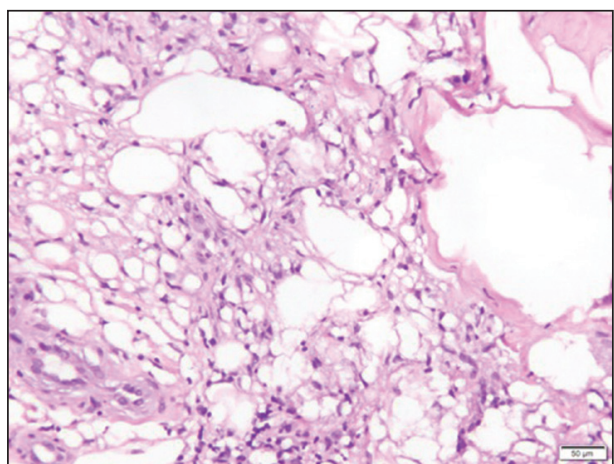

FIGURE 4. Lipodermatosclerosis. Membranous fat necrosis with cystic cavities lined by a hyaline membrane $(H \& E$, original magnification $\times 200)$. Reference bar indicates $50 \mu \mathrm{m}$.

characterized by membranous fat necrosis, fibrosis, and fatty microcysts that may be lined by a thickened hyaline membrane (Figure 4). Lipodermatosclerosis lesions generally do not resolve spontaneously and may need to be treated. ${ }^{16}$

\section{REFERENCES}

1. Musick SR, Lynch DT. Subcutaneous Panniculitis Like T-cell Lymphoma. StatPearls Publishing; 2020.

2. Guenova E, Schanz S, Hoetzenecker W, et al. Systemic corticosteroids for subcutaneous panniculitis-like T-cell lymphoma. $\mathrm{Br}$ J Dermatol. 2014;171:891-894.

3. Swerdlow SH. WHO Classification of Tumours of Haematopoietic and Lymphoid Tissues. International Agency for Research on Cancer; 2017.

4. Bagheri F, Cervellione KL, Delgado B, et al. An illustrative case of subcutaneous panniculitis-like T-cell lymphoma [published online March 3, 2011]. J Skin Cancer. doi:10.1155/2011/824528

5. Kogame T, Yamashita R, Hirata M, et al. Analysis of possible structures of inducible skin-associated lymphoid tissue in lupus erythematosus profundus. J Dermatol. 2018;45:1117-1121.

6. Arps DP, Patel RM. Lupus profundus (panniculitis): a potential mimic of subcutaneous panniculitis-like T-cell lymphoma. Arch Pathol Lab Med. 2013;137:1211-1215.

7. Alberti-Violetti S, Berti E. Lymphocytic lobular panniculitis: a diagnostic challenge. Dermatopathology. 2018;5:30-33.

8. Magro CM, Crowson AN, Kovatich AJ, et al. Lupus profundus, indeterminate lymphocytic lobular panniculitis and subcutaneous T-cell lymphoma: a spectrum of subcuticular T-cell lymphoid dyscrasia. J Cutan Pathol. 2001;28:235-247.

9. Sitthinamsuwan P, Pattanaprichakul P, Treetipsatit J, et al. Subcutaneous panniculitis-like T-cell lymphoma versus lupus erythematosus panniculitis: distinction by means of the periadipocytic cell proliferation index. Am J Dermatopathol. 2018;40:567-574.

10. LeBlanc RE, Tavallaee M, Kim YH, et al. Useful parameters for distinguishing subcutaneous panniculitis-like T-cell lymphoma from lupus erythematosus panniculitis. Am J Surg Pathol. 2016;40:745-754.

11. Burkholz KJ, Roberts CC, Lidner TK. Posttraumatic pseudolipoma (fat necrosis) mimicking atypical lipoma or liposarcoma on MRI. Radiol Case Rep. 2015;2:56-60.

12. Wick MR. Panniculitis: a summary. Semin Diagn Pathol. 2017;34:261-272.

13. Thurber S, Kohler S. Histopathologic spectrum of erythema nodosum. J Cutan Pathol. 2006;33:18-26.

14. Requena L, Requena C. Erythema nodosum. Dermatol Online J. 2002;8:4.

15. Choonhakarn C, Chaowattanapanit S, Julanon N. Lipodermatosclerosis: a clinicopathologic correlation. Int J Dermatol. 2016;55:303-308.

16. Huang TM, Lee JY. Lipodermatosclerosis: a clinicopathologic study of 17 cases and differential diagnosis from erythema nodosum. J Cutan Pathol. 2009;36:453-460. 